\title{
Metabolic evaluation guidelines in patients with nephrolithiasis: Are they being followed? Results of a national, multi-institutional quality assessment study
}

Sabrina S. Harmouch ${ }^{1}$; Hiba Abou-Haidar ${ }^{1}$; Hassan ElHawary²; Thomas Grgic ${ }^{3}$; Andrea G. Lantz $^{4}$; Jason Y. Lee ${ }^{5}$; Ben H. Chew ${ }^{3}$; Sero Andonian ${ }^{2}$; Naeem Bhojani ${ }^{1}$

${ }^{1}$ Division of Urology, University of Montreal Health Centre, Montreal, QC; ${ }^{2}$ Division of Urology, McGill University, Montreal, QC, Canada; ${ }^{3}$ Department of Urologic Sciences, University of British Columbia, Vancouver, BC; ${ }^{4}$ Department of Urology, Dalhousie University, Halifax, NS; ${ }^{5}$ Division of Urology, University of Toronto, Toronto, ON; Canada

Funding: Support for this project was awarded to Dr. Naeem Bhojani through the CUASF.

Cite as: Can Urol Assoc J 2018 May 28; Epub ahead of print. http://dx.doi.org/10.5489/cuaj.5155

Published online May 28, 2018

$* * *$

\section{Abstract}

Introduction: The significant cost burden of kidney stones underscores the importance of best clinical practice in kidney stone management. We evaluated adherence to kidney stone metabolic evaluation guidelines in a Canadian population and the interest of patients with regard to prevention.

Methods: A questionnaire based on Canadian Urological Association (CUA) best practice guidelines was designed. Patients presenting for extracorporeal shockwave lithotripsy treatment (ESWL) were administered this questionnaire to evaluate risk factors of stone disease and assess the use of metabolic evaluations. Patients were asked if they received explanations about their results and if they were interested in kidney stone prevention.

Results: We identified 530 patients at five academic institutions; 79.4\% had at least one indication to receive a metabolic evaluation (high-risk stone formers), which increased to 96.6\% if first-time stone formers that reported an interest in metabolic evaluation were included. However, only $41.1 \%$ of these patients had a metabolic evaluation. Endourologists ordered metabolic evaluation more often than other referring urologists (63.6\% vs. 36.5\%; $\mathrm{p}<0.001$ ). Furthermore, urologists ordered metabolic evaluations more often than other prescribing physicians (68.9\% vs. $31.1 \%$; $\mathrm{p}<0.001)$. Sixty-two percent of patients received explanations about their metabolic evaluation results and $77.5 \%$ 
understood them. Regarding prevention, $84.1 \%$ and $83.8 \%$ were interested in more explanations and in following a diet or taking a medication, respectively.

Conclusions: Adherence to CUA metabolic evaluation guidelines is suboptimal and could be improved by urologists referring patients for ESWL. Communication between physician and patient may not be adequate. The majority of stone formers are interested in kidney stone prevention.

\section{Introduction}

Renal stone disease has a lifetime occurrence risk of approximately $1 / 10$ and recent reports have shown that its incidence is rising.[1-4] Individuals who develop kidney stones have a $50 \%$ chance of recurrence within 5 years which adds to the cost burden to society associated with kidney stone disease estimated to be greater than $\$ 10$ billion dollars in 2006.[5-8] Moreover, the increasing prevalence of metabolic diseases such as obesity and diabetes and their association with stone disease are expected to increase the incidence of kidney stone disease and contribute to increasing the cost burden by 1.24 billion dollars per year by 2030.[9, 10] Similarly, the incidence of stone compositions related to dietary and metabolic risk factors, such as uric acid stones, is also believed to be increasing according to recent evidence.[9] Given the chronic nature of urolithiasis and the rising costs to the health care system, prevention of stone recurrence is important and highlights the potential value of following clinical practice guidelines in kidney stones management. Diet and lifestyle factors play a central role in kidney stone prevention and have the potential to slow down the epidemic of stone disease. As a matter of fact, level 1 evidence has shown that kidney stones are preventable.[11, 12] In particular, a 24-hour urinary metabolic evaluation and kidney stone analysis can assist in preventing recurrence by identifying modifiable risk factors for each individual patient which allows for a tailored approach, targeted at the patient's lithogenic risk factors.[12] Canadian Urological Association (CUA) Guidelines recommend that providers perform a metabolic evaluation in high-risk stone formers as well as in interested first-time stone formers.[12] The American Urological Association (AUA) and European Association of Urology (EAU) have similar guidelines.[13, 14] A previous publication has demonstrated that only $7.4 \%$ of patients with a kidney stone episode underwent metabolic evaluation in the United States.[15] However, adherence rates to CUA guidelines has not yet been examined in Canada. In light of this lack of evidence and concern regarding quality of care, this study aimed to evaluate the use of metabolic evaluations in a Canadian population, to assess the following: 1) adherence of referring physicians in ordering metabolic evaluations according to the CUA guidelines and 2) patients' understanding of their disease and their interest in following a diet or a treatment to prevent future stone events. The results of this study have implications for health care providers, stone formers and the Canadian healthcare system. 


\section{Methods}

Study population

A questionnaire based on current best practice guidelines for management of kidney stones according to the CUA (appendix 1) was designed. This questionnaire was reviewed by experts in the field of kidney stone disease and pre-tested among 15 patients on two consecutive occasions to reduce measurement bias. Patients who presented to participating academic centers for extracorporeal shock wave lithotripsy (ESWL) treatment between January and November 2017 were recruited to participate in this study and invited to fill out a short questionnaire that was available in English or French. The participating centers included the University of Montreal Health Centre (CHUM St-Luc), McGill University Health Centre (Glen Site), University of Toronto Kidney Stone Centre (St-Michael's Hospital), University of British Columbia Health Centre (Vancouver General Hospital) and the Queen Elizabeth II Health Sciences Centre (Halifax, NS). Patients with difficulties in communication (verbal, written and comprehension) in English or French, and those unwilling to participate voluntarily, were excluded from the study.

Questionnaire and characterization of patients

The questionnaire consisted of 4 parts and 16 questions in total. The first part of the questionnaire aimed to identify risk factors for stone disease (according to the CUA guidelines) by stratifying patients as high-risk of recurrence or first-time stone formers. Evaluated risk factors included medical history of multiple stone events, non-calcium stones, family history of stones, solitary kidney, chronic kidney disease, systemic disorders, gastro-intestinal disease, stones during pregnancy and occupations where public safety is at risk. Interested first-time stone formers were defined as patients, who were not at high-risk of stone recurrence (no risk factors) and who were interested in learning more about their disease. The second part of the questionnaire aimed to assess the use of metabolic evaluations for patients who had an indication to receive one according to the CUA guidelines by asking if patient had a 24-hour urine collection. The third part of the questionnaire aimed to assess if patients who received a metabolic evaluation received adequate explanations about the underlying cause of their kidney stone disease. Also, participants were asked if their physician explained their investigations results as well as their understanding of these explanations to assess for quality of communication between patient and physician. Finally, the fourth part of the questionnaire aimed to explore the patient's interest in kidney stone prevention by receiving more information about their disease or by following a diet or a specific treatment to prevent future stones events. The questionnaire also determined whether the use of metabolic evaluations varied according to the urologist's training (subspecialty in 
endourology), as patients were asked to specify their referring urologist. Anonymized data from all participating centers were recorded and pooled together for statistical analysis.

\section{Statistical analyses}

Sample size was initially calculated at 480 patients to estimate metabolic evaluation proportion with a level of confidence of $99 \%$, precision of $3 \%$, and expected proportion of $7 \%$ according to prior studies. Descriptive statistics and bivariate analyses such as cross-tabular analyses and chi-square test of association were performed to examine relationships between metabolic evaluation prescription patterns and referring physicians using IBM SPSS statistical software (SPSS version 24, New-York). All statistical analyses were two-sided and a p-value of $<0.05$ was considered statistically significant. Institution research ethics board (REB) approval was acquired in each participating center prior to conducting the study and informed consent was also obtained.

\section{Results}

Baseline characteristics

A total of 530 patients represented our study cohort and baseline characteristics are presented in Table 1. There were 358 participants from University of Montreal Health Centre, 51 from McGill University Health Centre, 43 from Saint Michael’s Hospital, 51 from Vancouver General Hospital and 27 from Queen Elizabeth II Health Sciences Centre. Median age was 56 years old (IQR: 46-63) and 58.7\% were male $(n=311)$ while $40.6 \%$ were female $(n=215) .45 .8 \%$ of referring urologists worked in a community setting ( $n=243), 40.0 \%(n=212)$ in an academic setting $(n=212), 14.9 \%$ of urologist were fellowship trained endourologists $(n=78)$ while $14.2 \%$ were unknown $(n=75)$.

Prevalence of risk factors and indications for metabolic evaluation

The majority of participants $(64.5 \%, n=342)$ had multiple or bilateral stones. $31.7 \%$ $(n=168)$ had a family history of stones, of which $83.9 \%$ (141) were first-degree relatives while $13.1 \%(n=22)$ were second-degree. Only $10.4 \%(n=55)$ of participants had a systemic disease and $4.3 \%(n=23)$ had either a solitary kidney or chronic kidney disease. $3.8 \%(n=20)$ of participants had stones during pregnancy and 3.2\% $(n=17)$ had a history of bariatric or intestinal surgery. Patients with an occupation associated with public safety (e.g. pilot) represented a small portion of the cohort $(1.3 \%, \mathrm{n}=7)$. Among all participants, $79.4 \%(n=421)$ had at least one indication to obtain a metabolic evaluation. When firsttime stone formers with an interest in getting a metabolic evaluation were included, $96.6 \%(n=512)$ of the study cohort should have gotten a metabolic evaluation (Figure 1 ). Only $21.1 \%$ of the study cohort stated they had their cause of stone disease explained to 
them, and the majority of causes explained to participants were related to genetic and dietary factors including low fluid intake.

Metabolic evaluation prescription patterns and interest in prevention

Among the 421 participants with at least one indication ( $\geq 1$ risk factor) to perform a metabolic evaluation (excluding interested first-time stone formers), less than half had one performed (41.8\%, $\mathrm{n}=176$ ) (Figure 1). Metabolic evaluations were more commonly performed when the referring urologist was an endourologist (63.6\%) compared to all other urologists (36.5\%) $(\mathrm{p}<0.001)$ (Figure 3). Moreover, these rates decreased when including interested first-time stone formers (34.3\% overall, $54.5 \%$ endourologist vs 29.5\% all other urologists) (Figure 1 and Figure 3). Less than two thirds of participants who received a metabolic evaluation reported getting an explanation about their investigations $(60.7 \%, \mathrm{n}=107)$ and $77.5 \%$ of these patients understood these explanations. The majority of providers of metabolic evaluations were urologists (68.9\%) followed by nephrologists (16.4\%) and family physicians (10.4\%). Approximately half of metabolic evaluations were performed within the last year, but of those metabolic evaluations done more than 1 year prior to ESWL, >20\% were done over 5 years prior to the current ESWL session. The majority of the study cohort (84\%) had an interest in having more information about their kidney stone disease and in following a diet or taking medications to prevent kidney stone recurrence.

\section{Discussion}

The present study carries several major findings. To the best of our knowledge, it is the first study to describe prevalence of risk factors of kidney stones, indication rates and metabolic evaluation of high-risk and interested first-time stone formers in an exclusively Canadian population.

First, we found that only $41.8 \%$ of high-risk stone formers ( $\geq 1$ risk factor) and $34.3 \%$ of high-risk as well as interested first-time stone formers ( $\geq 1$ risk factor or interest in metabolic evaluation) underwent a metabolic evaluation as recommended by the CUA guidelines (Figure 1). This insufficient use of metabolic evaluations was noted in both groups and these findings are similar to previous reports.[15, 16] For example, Gampsas et al. relied on a cohort of 199 first-time and recurrent stone formers who underwent ESWL during historical years (1987-1996) to examine the use of metabolic evaluations in kidney stone formers. These authors found a metabolic evaluation rate of $30 \%$ in highrisk stone formers and of $46 \%$ in high-risk as well as interested first-time stone formers.[16] Similarly, Milose et al. reported a suboptimal use of metabolic evaluations, using the Market Scan database. However, the prevalence rate (7\%) was almost 6 times lower than the one observed by Gampsas et al. and our group. These discrepancies can be explained by the differences in methodology as Milose et al. focused only on high-risk 
stone formers using a transversal retrospective design within a historical period (20022006). Unlike our analyses, baseline characteristics and prevalence of risk factors in their study cohort were not described. In consequence, differences in the distribution of these characteristics may have led to a lower estimate in their study cohort or a higher estimate in our study cohort. Likewise, recall bias may have overestimated our results.

Second, urologists with fellowship training in endourology were highly associated with the use of metabolic evaluations (Figure 3). Similarly, urologists and nephrologists also prescribed metabolic evaluations at a higher rate than family physicians. These findings are in agreement with previous studies and may be explained by several hypotheses such as more adequate knowledge, physician's beliefs as well as ability and comfort to take action on the results of the investigations.[15]

Third, our results showed low rates of explanations regarding metabolic evaluation results to patients by prescribing physician. Moreover, the reported comprehension of these explanations by patients were suboptimal. In addition, a small proportion of patients had the etiology of their kidney stones disease explained. Taken together, these observations suggest a lack of adequate physician-patient communications. and raise an important concern regarding quality of physician and patient dialog. However, these results could be explained by other factors. For example, it is possible that patients may be receiving the results of their metabolic evaluation at their post-ESWL treatment office visit. Also, another plausible explanation may be that patients do not have an identifiable etiology of their stone disease. Improving our knowledge of the pathophysiology of stone disease will help improve communication and prevention.

Fourth, we found that the majority of kidney stones formers were interested in kidney stone prevention by having more information about their disease and/or by following a diet or taking a medication for prevention of kidney stone events. Our findings are consistent with previous reports where interest in kidney stones prevention by kidney stones formers was examined.[16, 17] For example, Bensalah K. et al.'s observed that more than $80 \%$ of stone formers would consider preventive medical therapy to avoid recurrent pain or a surgical procedure related to kidney stones events.[17] Congruently, Gampsas et al. demonstrated that 95\% of recurrent and firsttime stone formers were interested in identifying the underlying cause of their stone disease and almost the entire study cohort were unanimously willing to undergo a preventive treatment (98\%).[16]

Finally, we observed that $50 \%$ of metabolic evaluations were performed more than 1 year prior to the current ESWL treatment. Furthermore, > 20\% were done more than 5 years since the current ESWL treatment. The recurrent nature of kidney stones and the ever-changing medical condition of patients should be evaluated when considering 
the use of a metabolic evaluation. Although sometimes difficult for patients, repeated metabolic evaluations are imperative to assure proper kidney stone prevention. They should be repeated annually or with greater frequency, depending on kidney stone activity and can help determine adherence to treatment and possible metabolic changes .[12, 18] Hence, the recurrent nature of kidney stones and the ever-changing medical condition of patients should be evaluated when considering the need for repeated metabolic evaluations.

Our study has numerous strengths, including multi-institution participation from eastern, central and western provinces in Canada and participation of a superior number than initially calculated sample size (99\% confidence interval, precision of 3\%). Also, referring urologists included community and academic urologists as well as inclusion of high-risk and interested first-time stone formers. Despite its strengths, this study should be considered in the context of important limitations. The validity of the questionnaire was not tested which is subject to measurement error bias. However, we aimed to minimize this common limitation by reviewing the questionnaire content with experts and performed a pretest period of the questionnaire prior to the study. Although data was obtained from retrospective self-reporting by patients and is inherent to recall bias, a 24hour urine collection seems to be a memorable event. Moreover, the number of patients who were approached and did not accept to participate in the study was not available due to logistical limitations of our multi-institutional study, lack of fiscal and personnel resources. Consequently, response rate and non-response bias could not be evaluated in our study. Furthermore, some patients may be offered a metabolic evaluation after their stone treatment, which may have underestimated the frequency of treating physician offering metabolic evaluations. In addition, some patients may be interested in investigating kidney stone cause but will not be compliant in submitting a 24-hour urine collection. Finally, the medical charts and imaging studies of patients were not reviewed. In consequence, it is possible that patients may have forgotten to mention other stonerelated risk factors or patients may not have been aware of the presence of multiple or bilateral kidney stones.

Notwithstanding these limitations, this study has several important implications and novel findings. To begin with, it is the first qualitative assessment study of adherence to metabolic evaluation in kidney stones formers in Canada using a contemporary population. These findings contribute to evidence of the quality of care concern in North America regarding metabolic evaluation in kidney stone formers that was first observed more than fifteen years ago. Moreover, the overwhelmingly high interest in kidney stone prevention through patient education as found in this study shows a clear opportunity to empower and involve patients in the management of their stone disease. Physicians should be encouraged to discuss and explore these topics during clinical visits. 
Additionally, further work should be conducted to identify barriers and causes of lack of adherence to metabolic evaluation guidelines by providers in order to propose and adopt optimal strategies to reverse this harmful finding.[19] For instance, education of physicians, medical aid tools, referral to stone specialists and tertiary stone clinics have been suggested to increase the use of metabolic evaluations. Moreover, stone prevention programs have been proven capable and efficient in several studies with the enrollment of stone formers, which has the potential to lead to the reduction of medical expenditures related to kidney stones disease.[19-23] Finally, the 2016 updated CUA guidelines still categorize metabolic evaluation as grade C Oxford levels of evidence while the 2014 EAU guidelines upgraded the level of evidence regarding metabolic evaluations in highrisk stone formers to a grade A recommendation.[12, 14, 24] Therefore, quantitative research, randomized clinical trials and cost-effectiveness studies of metabolic evaluations in high-risk and interested first-time stone formers may be warranted to reassess the level of evidence of metabolic evaluations.

The use of metabolic evaluations in kidney stone formers is suboptimal. Compared to the cost associated with surgical interventions and hospital admissions, the cost of a metabolic evaluation is marginal and its impact on health care expenditures can be significant. Building a sustainable health care system is a major concern of our society and improving quality of care by following best clinical practice guidelines in kidney stone management can contribute to it. 


\section{References}

1. Ghani, K.R., J.D. Sammon, N. Bhojani, et al., Trends in percutaneous nephrolithotomy use and outcomes in the United States. J Urol 2013. 190: p. 55864.

2. Leow, J.J., C.P. Meyer, Y. Wang, et al., Contemporary Trends in Utilization and Perioperative Outcomes of Percutaneous Nephrolithotomy in the United States from 2003 to 2014. J Endourol 2017. 31: p. 742-750.

3. Ghani, K.R., J.D. Sammon, P.I. Karakiewicz, et al., Trends in surgery for upper urinary tract calculi in the USA using the Nationwide Inpatient Sample: 19992009. BJU Int 2013. 112: p. 224-30.

4. Scales, C.D., Jr., A.C. Smith, H.J. M., et al., Prevalence of kidney stones in the United States. Eur Urol 2012. 62: p. 160-5.

5. Pearle, M.S., E.A. Calhoun, and G.C. Curhan, Urologic diseases in America project: urolithiasis. J Urol 2005. 173: p. 848-57.

6. Lotan, Y. and M.S. Pearle, Economics of Stone Management. Urol Clin North Am 2007. 34: p. 443-453.

7. Sutherland, J.W., J.H. Parks, and F.L. Coe, Recurrence after a single renal stone in a community practice. Miner Electrolyte Metab 1985. 11: p. 267-9.

8. Trinchieri, A., F. Ostini, R. Nespoli, et al., A prospective study of recurrence rate and risk factors for recurrence after a first renal stone. J Urol 1999. 162: p. 27-30.

9. Antonelli, J.A., N.M. Maalouf, M.S. Pearle, et al., Use of the National Health and Nutrition Examination Survey to calculate the impact of obesity and diabetes on cost and prevalence of urolithiasis in 2030. Eur Urol 2014. 66: p. 724-9.

10. Lotan, Y., J.A. Cadeddu, C.G. Roerhborn, et al., Cost-effectiveness of medical management strategies for nephrolithiasis. J Urol 2004. 172: p. 2275-81.

11. Fink, H.A., J.W. Akornor, P.S. Garimella, et al., Diet, fluid, or supplements for secondary prevention of nephrolithiasis: a systematic review and meta-analysis of randomized trials. Eur Urol 2009. 56: p. 72-80.

12. Dion, M., G. Ankawi, B. Chew, et al., CUA guideline on the evaluation and medical management of the kidney stone patient - 2016 update. Can Urol Assoc J 2016. 10: p. E347-E358.

13. Pearle, M.S., D.S. Goldfarb, D.G. Assimos, et al., Medical Management of Kidney Stones: AUA Guideline. J Urol 2014. 192: p. 316-324.

14. Skolarikos, A., M. Straub, T. Knoll, et al., Metabolic evaluation and recurrence prevention for urinary stone patients: EAU guidelines. Eur Urol 2015. 67: p. 75063. 
15. Milose, J.C., S.R. Kaufman, B.K. Hollenbeck, et al., Prevalence of 24-hour urine collection in high risk stone formers. J Urol 2014. 191: p. 376-80.

16. Grampsas, S.A., M. Moore, and P.S. Chandhoke, 10-year experience with extracorporeal shockwave lithotripsy in the state of Colorado. J Endourol 2000. 14: p. 711-4.

17. Bensalah, K., A. Tuncel, J.D. Raman, et al., How Physician and Patient Perceptions Differ Regarding Medical Management of Stone Disease. J Urol 2009. 182: p. 998-1004.

18. Pearle, M.S., D.S. Goldfarb, D.G. Assimos, et al., Medical management of kidney stones: AUA guideline. J Urol 2014. 192: p. 316-24.

19. NEHI. Improving Physician Adherence to Clinical Practice Guidelines - Barriers and strategies for change. February 2003 Accessed September 28, 2017]; Available from: http://www.nehi.net/publications/53-improving-physicianadherence-to-clinical-practice-guidelines/view.

20. Parks, J.H. and F.L. Coe, Evidence for durable kidney stone prevention over several decades. BJU Int 2009. 103: p. 1238-46.

21. Lingeman, J., H. Mardis, R. Kahnoski, et al., Medical reduction of stone risk in a network of treatment centers compared to a research clinic. J Urol 1998. 160: p. 1629-34.

22. Hosking, D.H., S.B. Erickson, C.J. Van den Berg, et al., The stone clinic effect in patients with idiopathic calcium urolithiasis. J Urol 1983. 130: p. 1115-8.

23. Parks, J.H. and F.L. Coe, The financial effects of kidney stone prevention. Kidney Int 1996. 50: p. 1706-12.

24. Howick, J., Levels of evidence. Oxford, UK: Oxford Centre for Evidence-based Medicine 2009. 
Figures and Tables

Fig. 1. Metabolic evaluation prescription patterns in a Canadian population of kidney stone formers $(n=530)$.

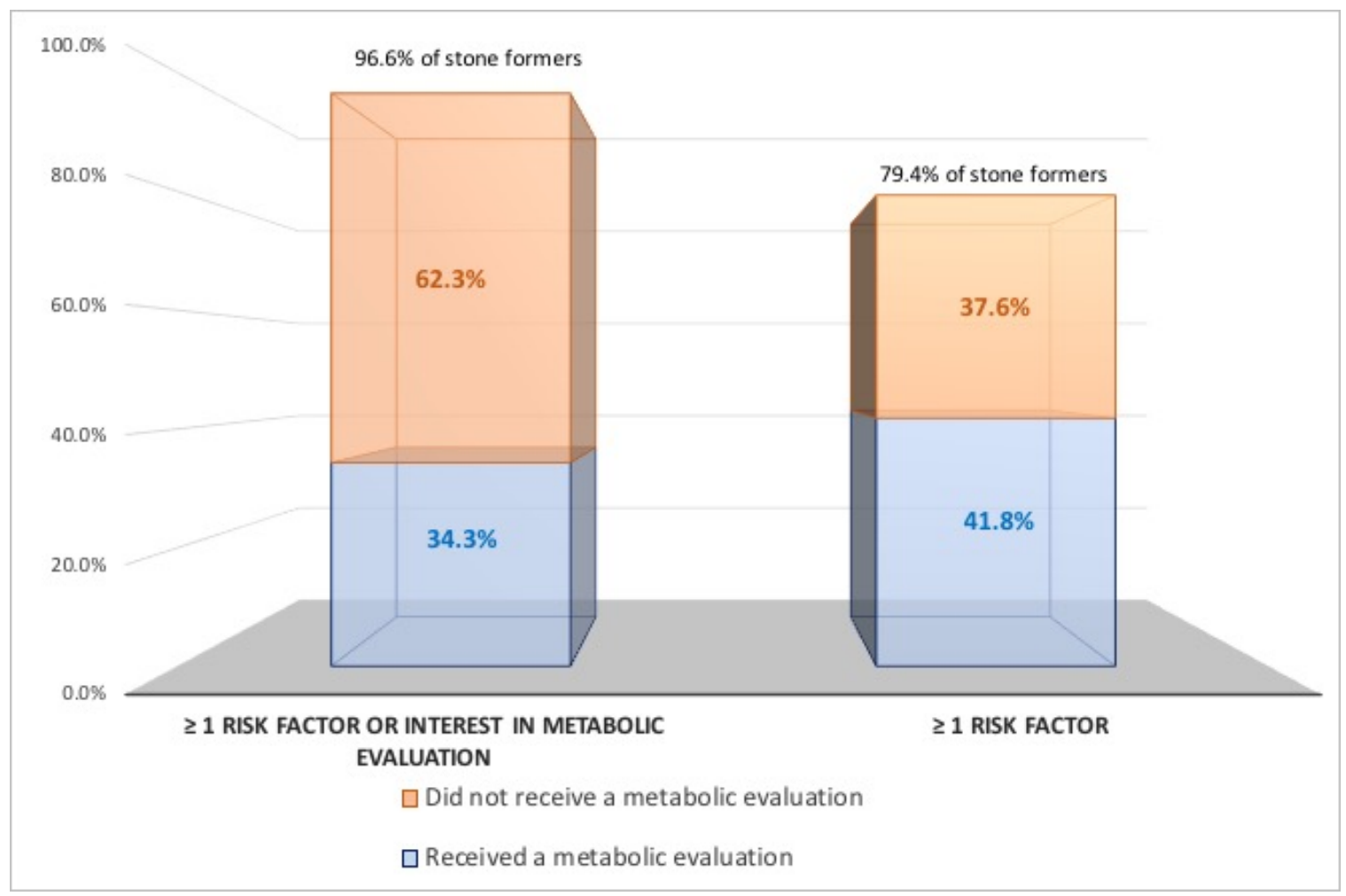


Fig. 2. Distribution of metabolic evaluations prescribing physicians.

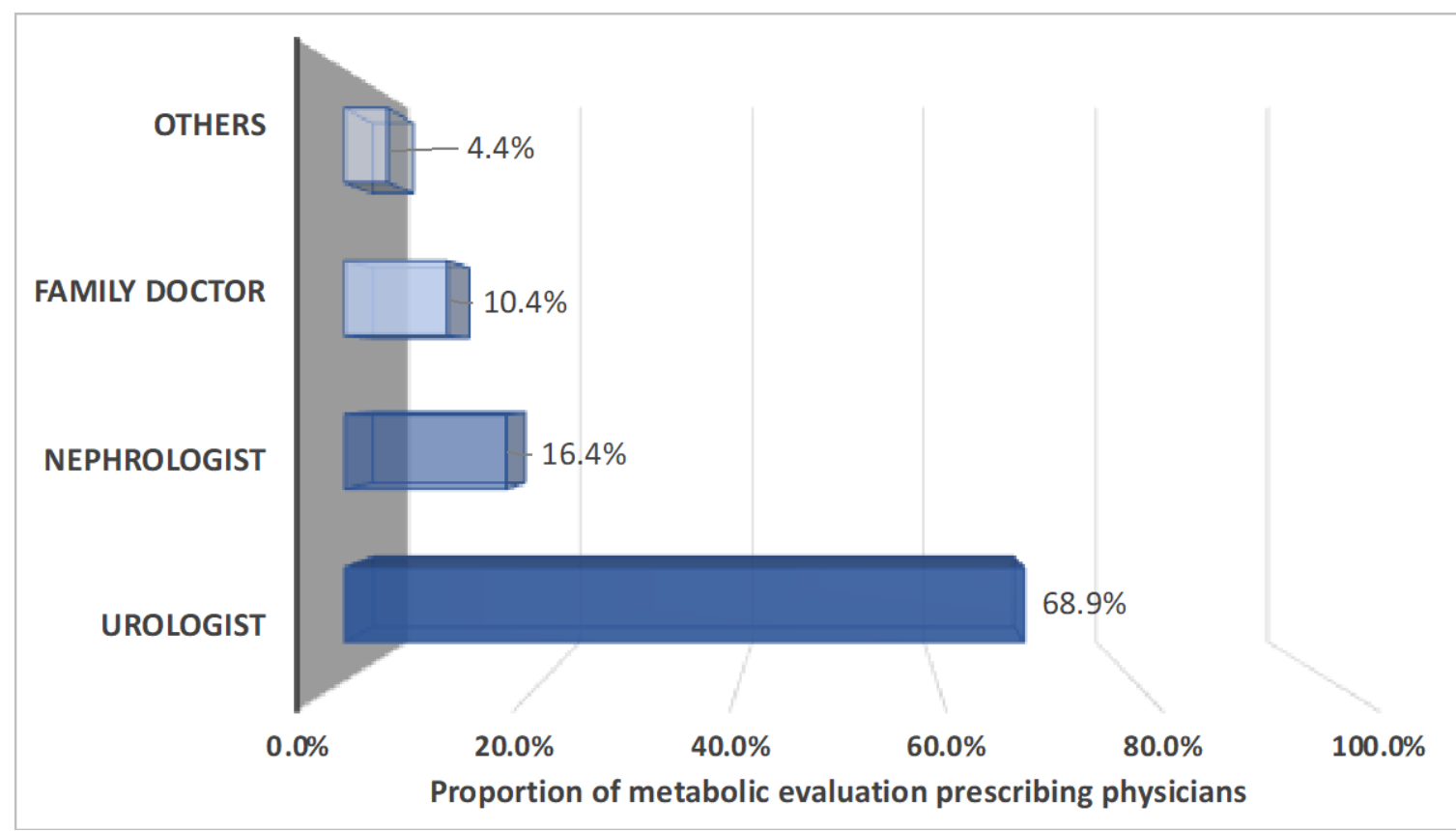


Fig. 3. Metabolic evaluation use according to urology training in a Canadian population of kidney stone formers $(n=530)$.

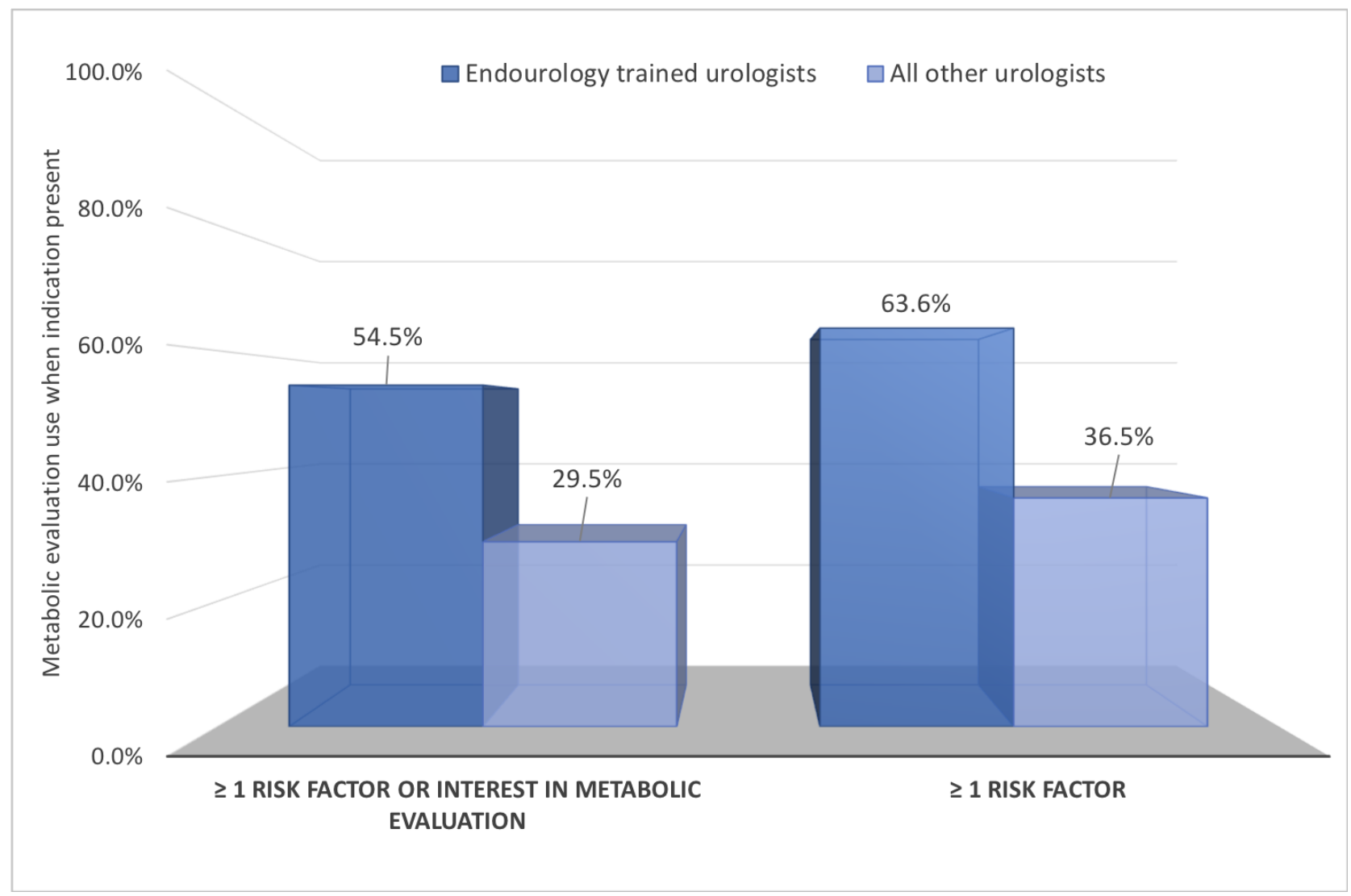




\begin{tabular}{|c|c|c|}
\hline Variables & $\%(n)$ & $\mathbf{p}$ \\
\hline Age, mean & 54.4 & \\
\hline \multicolumn{3}{|l|}{ Sex } \\
\hline Male & $58.7(311)$ & \\
\hline Female & $40.6(215)$ & \\
\hline Unknown & $0.8(4)$ & \\
\hline \multicolumn{3}{|l|}{ Referring urologists } \\
\hline In-community & $45.8(243)$ & \\
\hline Academic & $40.0(212)$ & \\
\hline Endourologists & $14.9(78)$ & \\
\hline Unknown & $14.2(75)$ & \\
\hline$\geq 1$ indication & $79.4(421)$ & \\
\hline$\geq 1$ indication including interested stone formers & $96.6(512)$ & \\
\hline \multicolumn{3}{|l|}{ Indications } \\
\hline Multiple/bilateral stones & $64.5(342)$ & \\
\hline Family history of stones & $31.7(168)$ & \\
\hline First-degree & $83.9(141)$ & \\
\hline Second-degree & $13.1(22)$ & \\
\hline Systemic disease & $10.4(55)$ & \\
\hline Single kidney/CKD & $4.3(23$ & \\
\hline Poor kidney function & $6.4(34)$ & \\
\hline Non-calcium stones & $4.3(23)$ & \\
\hline Stones during pregnancy & $3.8(20)$ & \\
\hline Bariatric or intestinal surgery & $3.2(17)$ & \\
\hline Occupation with risk for public safety & $1.3(7)$ & \\
\hline Cause of stone disease explained (\%) & $21.1(112)$ & \\
\hline Interested in more information about disease & $84.5(448)$ & \\
\hline Interested in following a diet or taking medications for disease & $83.8(444)$ & \\
\hline \multicolumn{3}{|l|}{ Metabolic evaluation } \\
\hline Received when indication $\geq 1$ indication present & $41.8(176)$ & \\
\hline Endourologists $(\mathrm{n}=66)$ & $63.6(42)$ & $<0.001$ \\
\hline All other urologists $(n=293)$ & $36.5(107)$ & \\
\hline Academic urologist $(n=54)$ & $60.0(32)$ & $<0.001$ \\
\hline Community urologist $(\mathrm{n}=81)$ & $40.0(32)$ & \\
\hline
\end{tabular}




\begin{tabular}{|l|c|c|} 
Received $\geq 1$ indication including interested stone formers & $34.3(176)$ & \\
Endourologists (n=77) & $54.5(42)$ & $<0.001$ \\
All other urologists (n=362) & $29.5(107)$ & \\
Academic urologist (n=56) & $61.1(34)$ & $<0.001$ \\
Community urologist (n=88) & $38.9(34)$ & \\
\hline Explanations received regarding metabolic evaluation & 60.7 & \\
\hline Explanations understood & 77.5 & \\
\hline Date of metabolic evaluation & $51.1(90)$ & \\
Within the last year & $24.4(43)$ & \\
Between 1 and 5 years ago & $10.2(18)$ & \\
Between 5 and 10 years ago & $11.9(21)$ & \\
More than 10 years ago & & \\
\hline Metabolic workup prescriber (\%) & 68.9 & \\
Urologist & 16.4 & \\
Nephrologist & 10.4 & \\
Family doctor & 4.4 & \\
Others & & \\
\hline
\end{tabular}

